EPV175/\#156 HOMOLOGOUS RECOMBINATION REPAIR GENES TESTING IN A COHORT OF APULIAN OVARIAN CANCERS PATIENTS IN THE ROUTINE DIAGNOSTIC PROCEDURE

${ }^{1} \mathrm{~A}$ Turchiano*, ${ }^{1} \mathrm{DC}$ Loconte, ${ }^{2} \mathrm{R}$ De Nola, ${ }^{3} \mathrm{~F}$ Arezzo, ${ }^{2} \mathrm{G}$ Chiarello, ${ }^{1} \mathrm{~A}$ Pantaleo, ${ }^{1} \mathrm{M}$ lacoviello, ${ }^{1} \mathrm{R}$ Bagnulo, ${ }^{4} \mathrm{~V}$ Loizzi, ${ }^{2} \mathrm{M}$ Marinaccio, ${ }^{2} \mathrm{E}$ Cicinelli, ${ }^{2} \mathrm{G}$ Cormio, ${ }^{1} \mathrm{~N}$ Resta. ${ }^{1}$ University of Bari 'Aldo Moro', Department of Biomedical Sciences and Human Oncology (dimo), Medical Genetics, Bari, Italy; ${ }^{2}$ University of Bari 'Aldo Moro', Department of Biomedical Science and Human Oncology, Gynecology and Obstetrics Section, Bari, Italy; ${ }^{3}$ Policlinico of Bary, Obstetrics and Gynecology Department, Bari, Italy; ${ }^{4}$ University of Bari 'Aldo Moro', Interdisciplinar Department of Medicine, Bari, Italy

10.1136/ijgc-2021-IGCS.246

Objectives Pathogenic variants in homologous recombination repair (HRR) genes other than BRCA1/2 have been associated with a high risk of ovarian cancer (OC). These findings might be useful for therapeutic procedures such as PARPi. In current clinical practice, the importance of genetic testing has increased, although it is generally limited to BRCA1/2. Herein, we investigated the mutational status of both BRCA1/2 and 5 HRR genes (BRIP1, RAD51C, RAD51D, PALB2 and, BARD1) in 79 unselected OC, thus evaluating the advantage of multigene panel testing in the daily clinical practice.

Methods We analyzed 79 epithelial OC samples by using an NGS custom multigene panel of the 5 HRR pathways genes, beyond the genetic routine BRCA1/2 testing.

Results Overall, 21 pathogenic variants (26\%) were detected. The majority $(21,5 \%)$ of participants displayed a deleterious mutation in BRCA $1 / 2$, whereas $5 \%$ harboured a pathogenic variant in one of the HRR genes. Additionally, there were 15 (19\%) uncertain significant variants (VUS), 5 of which occurred in BRCA1/2 and 10 of which involved at least one HRR gene. The assessment of germline mutational status showed that a little number of variants (3 pathogenic mutations in BRCA1/2 as well as 2 VUS in BRCA1 and RAD51D) were not detected in the corresponding blood sample. Notably, we unveiled 1 BRIP1 and 4 BRCA1/2 deleterious variants in the low-grade serous and endometroid histology, respectively.

Conclusions We demonstrated that the usage of a multigene panel, beyond BRCA1/2, improves the diagnostic yield in OC testing and it could produce clinically relevant results.

\section{EPV176/\#158 INVESTIGATING A FAMILY OF CANCER-TESTIS ANTIGENS AS BIOMARKERS FOR THE EARLIER DETECTION OF OVARIAN CANCER}

${ }^{1} \mathrm{~K}$ Grayson*, ${ }^{2} \mathrm{G}$ Khan, ${ }^{3} \mathrm{M}$ Hardman, ${ }^{4} \mathrm{~B}-\mathrm{A}$ Guinn. ${ }^{1}$ University of Hull, Biomedical Sciences, Hull, UK; ${ }^{2}$ Lancaster Medical School, Lancaster Medical School, Lancaster, UK; ${ }^{3}$ Hull York Medical School, Hull York Medical School, Hull, UK; ${ }^{4}$ University of Hull, Department of Biomedical Sciences, Hull, UK

\subsection{6/ijgc-2021-IGCS.247}

Objectives To investigate a family of cancer-testis antigens as biomarkers for early-stage ovarian cancer and whether they can be identified through non-invasive screening methods. We also aimed to examine the role of these cancer-testis antigens in disease progression.

Methods Manipulation of gene expression in ovarian cancer cell lines through plasmid and small interfering RNA transfection and immunocytochemistry of ovarian cancer stage I-IV tissue arrays.
Results Previously it has been shown that OCP2 is expressed at a significantly higher frequency in stage $I \quad(n=164)$ and II $(n=15)$ ovarian cancer tissue arrays than current clinically used biomarkers CA-125, HE4 and WT1. Analysis of ovarian cancer cell lines has shown that other family members, OCP3 and 4, are expressed at higher intensities than OCP2. Silencing of these genes in ovarian cancer cells lead to phenotypical changes followed by cell death observed within 24 hours. In addition, overexpression of these genes increases cell proliferation.

Conclusions This data provides a foundation for further investigation into OCPs as biomarkers for early-stage ovarian cancer in patient blood, urine and tissue. The small size of the proteins may allow them to be excreted and therefore applicable for non-invasive screening. The function of these proteins could also make them candidates for targeted immunotherapy.

\section{EPV177/\#165 IMPLEMENTATION OF ESGO QUALITY INDICATORS (QI) FOR OVARIAN CANCER SURGERY (OCS) IN A LATIN AMERICAN CENTER}

${ }^{1} \mathrm{~T}$ Silva*, ${ }^{2} \mathrm{~B}$ Ribeiro, ${ }^{3} \mathrm{~F}$ Schamne, ${ }^{4} \mathrm{R}$ Ribeiro, ${ }^{4} \mathrm{~J}$ Linhares, ${ }^{4,5} \mathrm{~A}$ Tsunoda. ${ }^{1}$ Hospital Erasto Gaertner, Ginecologic Oncology Fellow, Curitiba, Brazil; ${ }^{2}$ Hospital Erasto Gaertner, Surgical Oncology Resident, Curitiba, Brazil; ${ }^{3}$ Hospital Erasto Gaertner, Registered Research Nurse, Curitiba, Brazil; ${ }^{4}$ Hospital Erasto Gaertner, Surgical Oncology, Curitiba, Brazil; ${ }^{5}$ Pontificia Universidade Católica do Parana, Ppgts, Curitiba, Brazil

\subsection{6/ijgc-2021-IGCS.248}

Objectives To evaluate if the implementation of an institutional OCS protocol, aligned to NCCN guidelines, resulted in a high score index according to ESGO quality indicators (QI) in a Latin American public referral center.

Methods All consecutive surgical OC cases after a dedicated multidisciplinary team and protocol were instituted in 2015 up to 2018 were included. QI 1 to $10,2 \mathrm{y}$-DFS, 2y-OS and surgical complications were analyzed.

Results Ninety three patients, mean age $=59$ yo $(30-82$ yo), stage III $=44(47,3 \%)$ and $I V=20(21,5 \%)$, were included. QI 1-10 were $8,4,3,3,3,3,3,3,3,3$, sequentially. Debulking procedures were considered: CC0in 69 (65\%), CC1 in 6 (6\%), and CC2 in 26 cases (27.9\%). Complications, according to Clavien-Dindo, in 30 days, were minor in $2(2.1 \%)$ and major in 17 (18\%), including 3 post-operative deaths (3.2\%). Two-years DFS, relapsed, persistent and deaths were 41 (44.1\%), 16 (17.2\%), 10 (10.7\%), 26 (29\%), respectively.

Conclusions Adherance to ESGO QI was feasible and reproducible in a Latin American referral center. Similar criteria could be replicable in LMIC countries for OCS quality assurance.

\section{EPV178/\#166 IDENTIFICATION OF BIOMARKERS AND TARGETS FOR THE IMMUNOTHERAPY OF PATIENTS WITH CLEAR CELL OVARIAN CANCER: A SYSTEMATIC LITERATURE REVIEW}

${ }^{1}$ H Butler*, ${ }^{2} \mathrm{R}$ Glasspool, ${ }^{3}$ B-A Guinn. 'University of Hull, Hull York Medical School, Hull, UK; ${ }^{2}$ The Beatson Institute for Cancer Research, Clinical Trials Unit, Glasgow, UK; ${ }^{3}$ University of Hull, Department of Biomedical Sciences, Hull, UK

\subsection{6/ijgc-2021-IGCS.249}

Objectives Clear cell ovarian cancer (OC) is a rare type of epithelial cancer commonly associated with endometriosis. 